\title{
Symposium: Basic Science Committee: Latest Developments in Medical Imaging
}

\section{SY19-1107}

MRI of cartilage - Identification of early changes

G.H. Welsch $^{1}$, S. Domayer ${ }^{2}$, S. Apprich ${ }^{3}$, M. Blanke ${ }^{1}$, S. Marlovits ${ }^{4}$, S. Trattnig ${ }^{3}$

${ }^{1}$ Universitätsklinikum Erlangen, Trauma Surgery, Erlangen, Germany,

${ }^{2}$ Medical University of Vienna, Orthopedic Surgery, Vienna, Austria, ${ }^{3}$ Medical University of Vienna, Radiology, Vienna, Austria,

${ }^{4}$ Medizinische Universität Wien, Abt. Traumatologie, Wien, Austria Objectives: Besides the morphological description of cartilage repair tissue by means of MRI, different biochemical MRI techniques are seen to reflect different components of articular cartilage ultrastructure. Aim of this study was to exploit delayed gadolinium-enhanced MRI of cartilage (dGEMRIC), T2 mapping and diffusion weighted imaging (DWI) in a clinical approach in patients after cartilage repair: 1. to compare the ultrastructure of the repair tissue and native cartilage; 2. to correlate the biochemical parameters; 3 . to assess their relation to the clinical outcome.

Methods: Twenty patients (age: $34.7 \pm 9$ years, follow-up: $35.1 \pm$ 20 months) after matrix-associated autologous chondrocyte transplantation (MACT) of the knee were included at 3T MRI. Besides morphological MRI, dGEMRIC, T2 mapping and DWI sequences were accomplished. A semi-automated zonal region of interest analysis was performed in the cartilage repair tissue and the surrounding native cartilage. Clinical testing was performed by the Lysholm score and the international knee documentation committee (IKDC) score. Statistical analysis-of-variance and Pearson correlation was performed.

Results:

1. The repair tissue showed significant $(p<0.001)$ lower dGEMRIC values compared to the reference cartilage. Comparably,
DWI values showed significant $(p<0.001)$ higher values for the repair tissue compared to the control cartilage, whereas T2 mapping showed less differences $(p=0.003$ to $p=0.092)$.

2. The correlation of dGEMRIC and DWI was clearly significant (Pearson: $0.265-0.483$ ). Nearly no correlation could be assessed in between dGEMRIC and T2 mapping, whereas T2 mapping and DWI showed in parts significant correlations (Pearson: 0.208$0.415)$.

3. The correlation in between the clinical scores and the biochemical scores showed in many of the cases significant correlations, with moderate Pearson correlation coefficients in between 0.216 and 0.523 . The clearest correlation could be assessed in between DWI and the clinical outcome, whereas T2 mapping as well as dGEMRIC and the clinical scoring revealed lower, and in parts not significant correlation coefficients.

Conclusions: The results of the present study showed comparable results to existing in vitro and in vivo studies, however there is no study available reporting and comparing all three methodologies together in a clinical approach. Reflecting the composition of the repair tissue, dGEMRIC is seen to be sensitive for the glycosaminoglycan (GAG) content, T2 mapping for the collagen matrix and the hydration, and DWI for the diffusivity (intra- and extracellular borders) within the tissue.

In relation to the clinical results, the diffusivity might provide the most sensitive parameter for the present quality of the repair tissue, whereas GAG content and the collagen matrix might predict its future quality. Based on the present approach, in future clinical studies and trials, the composition/ultrastructure of the repair tissue can be quantified and assessed non-invasively. 